\title{
Road Traffic Management using VANET
}

\author{
Sonali S. Pawar ${ }^{1}$, Priyanka V. Chaudhari ${ }^{2}$, Archana B. Deokate ${ }^{3}$, Surendrapal P.Wankhede ${ }^{4}$ \\ Student, Computer Engineering, Genba Sopanrao Moze College of Engineering, Pune, India ${ }^{1,2,3,4}$
}

\begin{abstract}
With the constant increase in transport traffic, existing traffic management solutions have become inefficient. Urbanization has diode to a rise in traffic jams and accidents in major cities. So as to accommodate the growing wants of transport systems today, there's a desire for an Intelligent Transport System. Vehicular Adhoc Network (VANET) may be a growing technology that assists in Intelligent Transport Systems. Vanets modify communication between vehicles similarly as fastened infrastructure known as Road side Units (RSU). We tend to propose a distributed, cooperative traffic congestion detection and dissemination system that uses VANET. Every of the driver's sensible phones is equipped with a Traffic App that is capable of location detection through Geographic Position primarily based System (GPS). This info is relayed to a foreign server that detects traffic jam. Once congestion is confirmed the congestion info is disseminated to the tip user phone through rsus. The Mobile App transmits the placement info at periodic intervals. Victimization the latitude, line of longitude and the current time, the placement of every vehicle is traced. Victimization location info, the space moved by the vehicle at a given time is monitored. If the value is below a set threshold, congestion is suspected during a specific space. If several vehicles within the same space send similar messages, traffic jam is confirmed. Once traffic jam is confirmed, the vehicles approaching the engorged space area unit well-read about the traffic through show boards that area unit available within the nearest rsus (traffic signals). The congestion info is additionally created accessible through the Mobile App gift in vehicles approaching the engorged space. The approaching vehicles might take diversion and alleviate congestion.
\end{abstract}

Keywords: Provenance Model, Security, Sensor Networks, Bloom Filter Packet generation, Fault location.

\section{INTRODUCTION}

Transportation is an activity involving the movement of people or product from one place to a different so as to meet the perceived social and economic desires of a user. As these desires amendment, the transportation system itself evolves and issues occur because it becomes troublesome to serve the general public interest. One of the negative impacts of any facility is traffic congestion [1].Vehicular traffic congestion may be a condition that happens on road networks, which involves the exaggerated queuing of vehicles characterized by exaggerated trip times and fuel consumption. Holdup happens where demand exceeds the capability of the transportation system and therefore the commonest drawback is queuing up of vehicles close to toll booths [2]. Traffic will be witnessed primarily on highways and major roads that connect industries. Metropolitan and cosmopolitan cities square measure found to own higher traffic congestion than alternative components. Traffic congestion poses varied threats and arises numerous issues to the vehicle drivers. Various industries that bank on freight transport lose a significant sum of cash annually attributable to holdup. Various establishments, government bodies and organizations have undertaken surveys and studies tounderstand the causes and consequences of conveyance traffic congestion and conjointly to produce economical solutions to avoid holdup. VANET also helps USA to realize automobile to automobile communications and perform simulations at a comparatively low value [3]. Recent efforts have placed a robust stress on VANET style implementations [4].

\section{MOTIVATION}

The steady population increase in urban areas has led to a exponential increase in the number of vehicles on road. Vehicular traffic is one of the most important social and economic issues faced today resulting in congestion. With tremendous growth in industries, the need to reach the destination within a certain time is on-demand. Problem occurs when important destinations lie on the same route. A single Technology park housing multiple companies with Each company accommodating more than a hundred Employees is one such example. In these cases, traffic becomes unavoidable and there is a need for a Solution to avoid vehicular traffic congestion. A smart transport system which will provide real time information about the traffic by $\mathrm{p} 2 \mathrm{p}$ [5] is the need of the hour. The existing smart transport systems demand a need for the construction of expensive infrastructures or a change in the road structure. Although these systems prove to be very effective, they will consume enormous amount of time and cost to be deployed. Nowadays, the roads within cities are well-connected and therefore there are numerous routes for a single destination. If one route is congested, an alternate route can be taken. A simple solution to traffic congestion is to gain knowledge about the traffic congested routes. Once this knowledge is gained, it is feasible to identify an alternate path and divert 
from traffic congested areas. This solution not only helps in vehicle diversion but also resolves traffic congestion at a minimal time.

\section{III.LITERATURE REVIEW}

\section{MEASURING URBAN TRAFFIC CONGESTION - A REVIEW}

Traffic congestion has been one amongst major problems that almost all metropolises face. It's believed that identification of congestion is that the beginning for choosing applicable mitigation measures. Congestion - each in perception and actually - impacts the movement of individuals traffic jam wastes time, energy and causes pollution. There square measure generally 2 factors, that impact the congestion; (a) micro-level factors (b) macro-level factors that relate to overall demand for road use. Congestion is 'triggered' at the 'micro' level (e.g. On the road), and 'driven' at the 'macro' level. The small level factors square measure, for instance, many folks need to manufacturer at identical time, too several vehicles for restricted road area. On the opposite aspect, macro level factors square measure e.g. Landuse patterns, automobile possession trends, regional economic dynamics, etc. This paper offers an outline and presents the attainable ways that to spot and live metrics for urban blood vessel congestion. A scientific review is disbursed, supported menstruation metrics like speed, travel time/delay and volume and level of service. We have a tendency to review covers distinct aspects like definition; measuring criteria followed by totally different countries/organizations. Strengths and weaknesses of those measures square measure mentioned. Further, a brief critique of measurement criteria is presented.

\section{Traffic Congestion on Highways in Nigeria Causes, Effects and Remedies:}

Traffic congestion has been one amongst major problems that almost all metropolises face. It's believed that identification of congestion is that the beginning for choosing applicable mitigation measures. Congestion - each in perception and actually - impacts the movement of individuals traffic jam wastes time, energy and causes pollution. There square measure generally 2 factors that impact the congestion; (a) micro-level factors (b) macro-level factors that relate to overall demand for road use. Congestion is 'triggered' at the 'micro' level (e.g. on the road), and 'driven' at the 'macro' level. The small level factors square measure, for instance, many folks need to maneuver at identical time, too several vehicles for restricted road area. On the opposite aspect, macro level factors square measure e.g. Land-use, automobile possession trends, regional economic dynamics, etc. This Paper offers an outline and presents the attainable ways that to spot and live metrics for urban blood vessel congestion. A scientific review is disbursed, supported mensuration metrics like speed, travel time/delay and volume and level of service. We have a tendency to review covers distinct aspects like definition; measuring criteria followed by totally different countries/organizations. Strengths and weaknesses of those measures square measure mentioned. Further, a brief critique of measurement criteria is presented.

\section{Road Traffic Congestion in the Developing World:}

Road traffic jams still stay a serious drawback in most cities round the world, particularly in developing regions leading to large delays, hyperbolic fuel wastage and financial losses. Owing to the poorly planned road networks, a standard outcome in several developing regions is that the presence of tiny vital area unitas that are common hot-spots for congestion; poor traffic management around these hotspots probably leads to elongateed traffic jams. During this paper, we have a tendency to 1st gift an easy machine-controlled image process mechanism for detection the congestion levels in road traffic by process CCTV camera image feeds. Our algorithmic program is specifically designed for uproarious traffic feeds with poor image quality. Supported live CCTV camera feeds from multiple traffic signals in African country and Brazil, we have a tendency to show proof of this congestion collapse behaviour lasting long time-periods across multiple locations. To part alleviate this drawback, we have a tendency to gift space|a neighborhood decongestion protocol that coordinates stoplight behavior at intervals alittle area and may regionally forestall congestion collapse sustaining time variant traffic bursts. Supported a simulation based mostly analysis on easy network topologies, we have a tendency to show that our native de-congestion protocol will enhance road capability and stop congestion collapse in localized settings.

\section{Vanet Based Traffic Management System Development And Testing Using Aodv Routing Protocol:}

Vehicular Ad-Hoc network (VANET) may be a variety of Mobile Ad-Hoc (MANET) network wherever the nodes area unit unnatural to maneuver on the road. Vehicles in VANET area unit equipped with a radio device to speak with one another and additionally with the road facet units (base stations). Conveyance networks aims to create the driving expertise safer, economical and pleasant. Vehicular traffic congestion is mirrored as delays whereas traveling. Holdup contains a variety of negative effects and may be a major drawback in today's society. Many techniques are deployed to contend with this drawback. During this paper, it's been projected AN innovative approach to contend with the matter of holdup victimisation the characteristics of conveyance ad-hoc networks (VANET). The system is developed and tested victimisation AODV protocol of unintended mobile network to contend with the matter of car holdup in 
conveyance networks. The performance is measured in terms of no. Of packets broadcasted, proportion of packets delivered, and proportion of traffic entertained and overhead to manage the matter of information holdup in pc networks. Victimisation simulations the relevancy of the formula within the domain of car holdup during a VANET is incontestible.

\section{An Intelligent Traffic Light Control Approach for Reducing Vehicles CO2 Emissions in VANET:}

This paper proposes associate intelligent stoplight control theme to cut back vehicles dioxide emissions supported VANET. Among the planned theme, period traffic information will be obtained by wireless communication between the vehicles and also the traffic lights. The intelligent traffic light management approach will be rotten into 2 steps. First, associate adaptational stoplight management algorithmic program is proposed with purpose of reducing vehicles waiting time. We model four totally different phases, and style the demand perform To allot the inexperienced lightweight for every phases supported multiple traffic factors like traffic density etc. So as to decrease vehicles stop times, we have a tendency to then derive recommendatory speed for every individual vehicle. This step is to maximise the turnout of the intersection so the vehicle stopped by the sunshine will be Minimized. Additionally, we have a tendency to introduce a dioxide emissionestimation model to calculate vehicles dioxide emissions. Finally, comparing with pre-timed management methodology, simulation results Indicate that the planned theme will expeditiously reducing vehicle's average waiting time, stop times and dioxide emissions

\section{IV.NEED FOR SYSTEM}

A smart transport system which is able to give real time info concerning the traffic by $p 2 p$ is that the would like of the hour. The present smart transport systems demand a requirement for the development of high-priced infrastructures or a modification within the road structure. Though these systems persuade be terribly effective, they'll consume monumental quantity of your time and value to be deployed. Nowadays, the roads inside cities are connected and so there are various routes for one destination. If one route is full, an alternate route is taken. A straightforward answer to holdup is to realize data concerning the traffic full routes. Once this information is gained, it's possible to spot AN alternate path and divert from traffic full areas. This answer not solely helps in vehicle diversion however additionally resolves holdup at a least time.

\section{VEHICULAR ADHOC NETWORKS}

Currently, traffic data systems are centralized vehicular applications victimization technologies like Traffic Message Channel (TMC), provides data regarding road traffic conditions. However, it lacks short delay times (due to the centralized approach), averages data for giant geographical areas (due to cost-sensitiveness of elaborate detector networks and restricted radio resources) and doesn't have the chance to supply services for time vital applications. Such systems would, for instance, not meet the equirements of a congestion rejection application, as a result of they need long delays and would need giant capability owing to the massive region of service. In distinction, VANET-based systems will have short delays and also the capability may be reused additional expeditiously. Moreover, the structure of VANET may be Distributed, that improves the amount of independence, quantifiability and stability. VANET may be a quick growing and up to date technology to automate and ease transport problems like traffic, pollution, etc. During this paper, traffic jam management victimization VANET is achieved by setting up a Vehicle-InfrastructureVehicle network .These networks permit many applications, such as traffic safety, driver help and diversion for passengers. The constant movement of vehicles at varied speeds causes constant changes within the network topology. Further, vehicles have a restricted time to exchange information among alternative vehicles or the Road aspect Units (RSU). IEEE planned a family of standards for transport networks known as WAVE (Wireless Access within the transport Environment).

WAVE consists by 2 classes of standards:

(i) $802.11 \mathrm{p}$ for PHY and raincoat layers and (ii) IEEE 1609 for security, network management likewise as Other aspects of vanets

\section{PROPOSED SYSTEM}

The traffic management system consists of a Traffic App put in within the driver's smart phone and a distant server. Fig.1 illustrates the traffic management system. The GPS within the driver's smart phone detects the situation of the vehicle. The Traffic App extracts this location data and sends it Periodically to a distant server. The situation information consists of the latitude, longitude, and Date and time which might be extracted through a code This location data obtained from the sensible Phone is logged into a information sporadically mistreatment GPRS. This 
location data is employed to calculate traffic congestion. Traffic congestion is calculated by considering the location of the vehicle and also the range of vehicles in a particular location boundary. If a vehicle is found to. Transmit a similar location data for a prolonged amount of your time, then the vehicle is marked for traffic boundary. A traffic boundary is computed with the marked vehicle's location. The quantity of vehicles among that boundary is calculable. Each of these vehicles is checked for location to spot the density of traffic. The congestion data provides data concerning the existence of congestion during a explicit space. The congestion data is shipped to the sensible phones equipped with the Traffic App. By mistreatment this data, the vehicles will divert in alternate route. A diagram of the system is illustrated in Fig.2. Thus, a vehicle-infrastructure-vehicle communication system is expeditiously accustomed relay traffic info. However, whereas developing the system, steps should be taken to confirm that traffic information doesn't get superannuated. As traffic information is incredibly sensitive and significant, a frequent update on the traffic info is of utmost importance. Being a smart system, it may also analyze traffic in curvilinear roads and roads of lowest safety

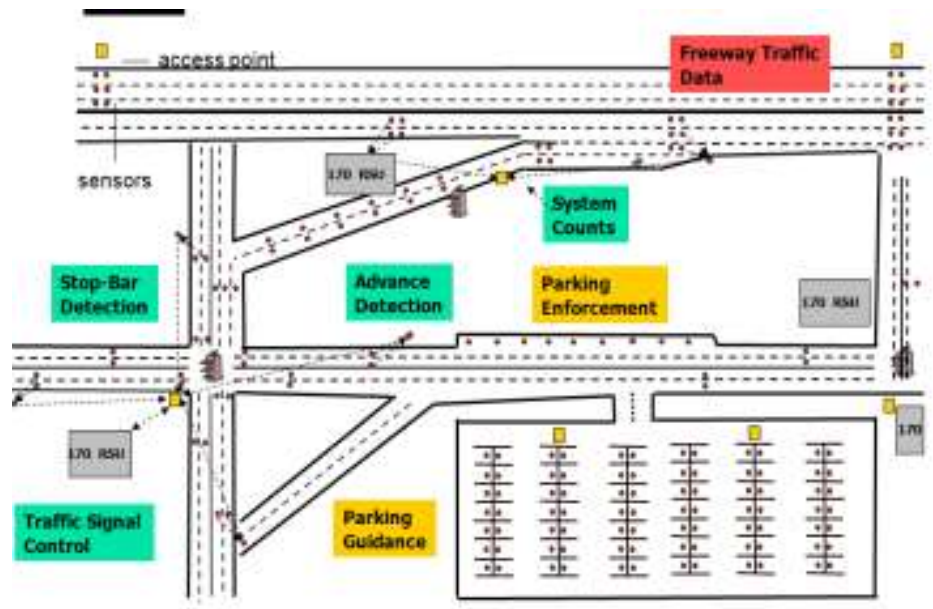

Fig 1. Architecture Diagram

\section{IMPLEMENTATION}

The entire traffic congestion management system Consists of 3 modules: (i) information Acquisition Module (ii) Congestion Detection Module and (iii) Congestion Dissemination Module.

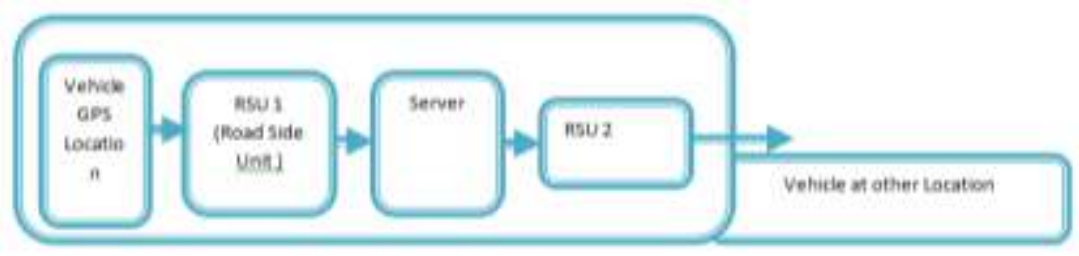

Fig 2. Block Diagram

\section{Data Acquisition Module:}

The Traffic App is put in within the driver's sensible Phone and accustomed get traffic info. The Traffic App has been developed within the automaton platform. Once this app is initialized, it permits the placement sensor of the smart phone. Fig.4 illustrates the Traffic App put in within the driver's smart phone. The "show location" button gift within the Traffic App gains access to the placement device of the smart phone and extracts the latitude and meridian of the device on with Greenwich time unit. This info is updated each fifteen seconds. On clicking the submit button within the app, it connects itself to the net Fi using the GPRS within the smart phone and logs the location info into the remote server. On clicking the "Get Traffic" button, the congestion information computed at the remote server is extracted and displayed for the motive force. The location info is keep in a web Database that consists of a table named location. The location table shown in Fig.3 stores the latitude, longitude, time and device ID received from varied mobile phones that are connected to the network. The database is reinvigorated each fifteen seconds to log the incoming values. The placement info during this database is employed by the congestion detection module to reason the congestion info

\section{Congestion detection Module:}

The system uses PHP for secret writing the congestion detection module. The location of this vehicle is extracted and a boundary of $100 \mathrm{~m}$ about is created around this vehicle. All different vehicles lying at intervals this boundary area unit 
checked for congestion. If 2 or a lot of vehicles area unit found to be below congestion, traffic congestion is asserted. Vehicles will perform lay vehicular communicate if they're at intervals a threshold distance

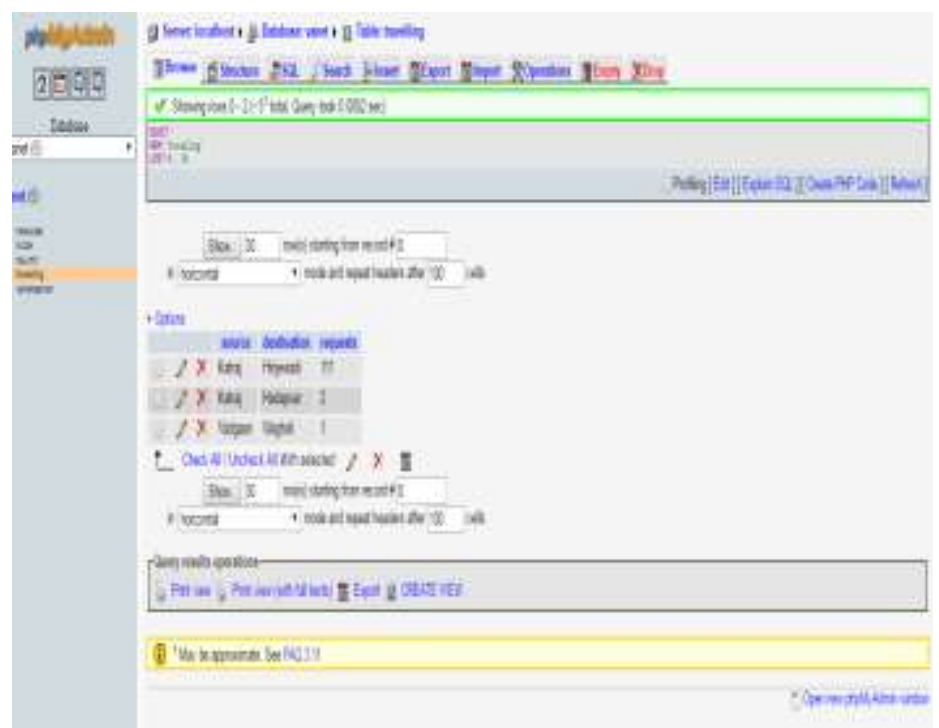

Fig 1. Wamp Server Localhost Database

\section{Congestion dissemination Module:}

The congestion data is transmitted to the Traffic App within the driver's smart phones that area unit approaching the actual engorged region. The congestion data is additionally transmitted to the rsus set within the close traffic signals. These rsus comprises liquid show (LCD) that displays congestion data. This information are often employed by the approaching vehicle drivers and that they will take alternate routes.

\section{CONCLUSION}

By exploitation the periodic congestion info, a congestion constant can be computed. A congestion constant could be a threshold worth that indicates the extent of traffic. The congestion coefficient price will vary between 0 to 10 with 0 indicating lowest congestion and 10 indicating highest congestion. So as to reason the coefficient, a study on the traffic atterns should be done. For every style of road, the factors that determine traffic are to be studied.

\section{ACKNOWLEDGMENT}

The heading of the Acknowledgment section and to boot the References section mustn't be numbered. It offers us nice pleasure in presenting the preliminary project report on "ROAD TRAFFIC MANAGEMENT victimisation VANET". I might need to want this chance to impart my internal guide for giving me all the assistance and steering I required. I'm really grateful to them for his or her kind support. Their valuable suggestions were really useful. I'm in addition grateful to Head of COMPUTER ENGINEERING Department for his indispensable support, suggestions. At intervals the top our special as a result of fully completely different Person Name for providing varied resources like laboratory with all required software system platforms, continuous web association, for Our Project.

\section{REFERENCES}

[1] Akhtar, Naheed, Sinem Coleri Ergen, and Oznur Ozkasap. "Vehicle mobility and communication Channel models for realistic and efficient highway VANET simulation." Vehicular Technology, IEEE Transactions on 64.1 (2015): 248-262.

[2] Cavalcante, Evellyn S., André LL Aquino, Gisele L. Pappa, and Antonio AF Loureiro. "Roadside unit deployment for information Dissemination in a VANET: An evolutionary Approach." In Proceedings of the 14th annual Conference companion on Genetic and Evolutionary computation, pp. 27-34. ACM, 2012.

[3] Gamati, Emadeddin A., Richard Germon, and Evtim Peytchev. "Collaborative Data Dissemination Methods In vanets For Identifying Road Conditions Zone Boundaries." In ECMS, pp. 372-378. 2013.

[4] Kausar, Tanwee, Priyanka Gupta, Deepesh Arora, and Rishabh Kumar. "A VANET based Cooperative Collision Avoidance System for a 4Lane Highway."

[5] Wang, Miao, et al. "Real-time path planning Based on hybrid-VANET-enhanced transportation System." Vehicular Technology, IEEE Transactions On 64.5 (2015): 1664-1678.

[6] He, Zongjian, Buyang Cao, and Yan Liu. "Accurate Real-Time Traffic Speed Estimation Using Infrastructure-Free Vehicular Networks." International Journal of Distributed Sensor Networks 2015 (2015). 